\title{
Low-complexity Frequency Synchronization for GSM Systems: Algorithms and Implementation
}

\author{
Harald Kröll*, Stefan Zwicky*, Christian Benkeser*, Qiuting Huang*, Andreas Burg ${ }^{\dagger}$ \\ *Integrated Systems Laboratory \\ ETH Zurich, 8092 Zurich, Switzerland \\ e-mail: \{kroell,zwicky,benkeser,huang\}@iis.ee.ethz.ch \\ ${ }^{\dagger}$ Telecommunications Circuits Laboratory \\ EPFL Lausanne, 1015 Lausanne, Switzerland \\ e-mail: andreas.burg@epfl.ch
}

\begin{abstract}
Carrier frequency synchronization of a mobile station (MS) is a crucial task at the beginning of the cell selection procedure and during normal operation. Constant monitoring of the base-stations surrounding the MS requires robust synchronization concepts with low computational complexity. In the GSM standard, periodically transmitted frequency correction bursts (FB) provide the necessary information for synchronization. This paper presents a combined low-complexity approach for FB detection and carrier frequency estimation, which achieves high detection probability and frequency estimation accuracy. A thorough performance assessment on a GSM/EGPRS2 testbed proves the suitability of the corresponding FPGA hardware implementation.
\end{abstract}

\section{INTRODUCTION}

In the past 20 years, the success story of the Global System for Mobile Communication (GSM) has led to more than 4 billion subscribers worldwide in 2010 [1]. In industrialized and newly-industrialized countries GSM builds a reliable fall-back solution for modern $3 \mathrm{G}$ and $4 \mathrm{G}$ systems, whereas in many other parts of the world GSM is the only cellular network available. With the latest standard extension EGRPS2, GSM can provide data rates of up to $1.2 \mathrm{Mb} / \mathrm{s}$ by introducing new technical features, such as 16QAM and 32QAM modulation.

The detection of higher order modulation EGPRS2 signals at acceptable SNR-levels requires high-end RF transceiver ICs with very low noise figure [2], and sophisticated channel equalization and demodulation algorithms in the digital baseband [3]. In legacy GSM modes with GMSK modulation the dramatic improvement of receiver sensitivity allows the operation at low SNR-levels, e.g., [2] with an input signal power level of $-110 \mathrm{dBm}$ which translates to an SNR of about $7 \mathrm{~dB}^{1}$. In order to maintain the low-cost attribute of $2 \mathrm{G}$ devices, low-complexity solutions that enable communication at such low SNR-levels are required for the fundamental problems in the digital baseband of GSM-based receivers.

In particular, an accurate time base is crucial in GSM receivers, however, the need for low-cost devices in mobile

\footnotetext{
${ }^{1}$ The noise figure is given with $\mathrm{NF} \geq 2.4 \mathrm{~dB}$ and we assume another $1.5 \mathrm{~dB}$ loss due to switching and SAW-filter between antenna and transceiver IC. The receiver sensitivity is typically given for a static one-tap channel profile.
}

stations (MS) prohibits the use of high quality oscillators. Typical digitally-controlled crystal oscillators (DCXO) in modern MSs can suffer from frequency inaccuracies of 20 parts per million (ppm). Calibration after production improves the oscillator accuracy to $5-10 \mathrm{ppm}$, which results in a maximum GSM carrier frequency offset of $f_{\mathrm{o}}= \pm 18 \mathrm{kHz}^{2}$. Before a MS can establish a connection to the base-station (BTS), the frequency offset has to be estimated accurately $\left(f_{\mathrm{o}} \leq 0.1 \mathrm{ppm}\right.$ after synchronization [4]), and corrected by tuning the oscillator of the MS.

In the GSM system, BCCH (Broadcast Control Channel) carriers provide the necessary information for synchronization. When the MS is switched on, the $\mathrm{BCCH}$ is monitored to detect the FB, a complex sinusoid with a frequency of $67.7 \mathrm{kHz}$ above the carrier frequency. The tasks to achieve frequency synchronization are 1) the detection of a FB, and then 2) the estimation of the carrier frequency offset by means of the FB's complex sinusoid. After successful detection and offset estimation, the local oscillator of the MS is tuned in order to establish frequency synchronization. Constant monitoring of the BTSs surrounding the MS requires FB detection (FBD) and frequency offset estimation (FOE) during normal operation.

Contributions and Outline: In this paper we present a joint approach for FBD and FOE for GSM. The parameters of the algorithms have been optimized to achieve excellent performance figures. Our proposed solution allows both FBD and FOE to be performed on one single FB to minimize synchronization time without expensive buffering. In Section II we introduce the system model. Then, in Section III we compare the most promising algorithms suitable for FBD in GSM, and propose a low-complexity solution, which achieves high detection probability at low false alarm rate. The corresponding FOE is presented in Section IV where we analyze algorithms that can be combined with FBD to minimize implementation complexity. We propose a frequency estimator based on Prony's method [5] that re-uses results of the FB detector to achieve high FOE accuracy at low computational cost.

\footnotetext{
${ }^{2}$ Most GSM networks operate with carrier frequencies around $900 \mathrm{MHz}$ or $1800 \mathrm{MHz}$. For the computation of the worst-case frequency offset we assumed a carrier frequency of $1800 \mathrm{MHz}$.
} 
Finally, the paper describes the performance assessment of the algorithms mapped to an FPGA on a GSM/EGPRS2 testbed. The measurement results in Section $\mathrm{V}$ prove the suitability of the proposed concepts for real-world applications.

\section{SySTEM MODEL}

In GSM and it's evolved EGPRS standards, BCCH carriers provide the necessary information for synchronization. Every 10th or 11th TDMA frame on a BCCH carrier contains a FB on time slot $\# 0$, i.e. the time period between two consecutive FBs is $46.2 \mathrm{~ms}$ or $50.8 \mathrm{~ms}$ respectively. FBs contain a stream of $N_{\mathrm{TOT}}=142$ consecutive logical zeros as data bits. With differential encoding and GMSK modulation two identical subsequent data bits cause a phase change of $+\pi / 2$ while two different symbols result in a phase change of $-\pi / 2$. This results in a sinusoid with frequency $f_{\text {sym }} / 4=67.7 \mathrm{kHz}$ above the carrier frequency, where $f_{\text {sym }}$ is the GSM symbol rate of $270.8 \mathrm{ksym} / \mathrm{s}$. We denote the discrete time received baseband signal of a FB as follows:

$$
y[n]=e^{j 2 \pi n\left(\frac{f_{\mathrm{sym}}}{4}+f_{\mathrm{o}}\right)} \star h[n]+w[n],
$$

where $h[n]$ denotes the complex baseband response of the channel and $f_{\mathrm{o}}$ the positive or negative frequency offset of the local oscillator in the receiver. $w[n]$ is complex additive white Gaussian noise (AWGN) and $\star$ the convolution operator. Frequency synchronization in GSM requires the detection of the FB sequence on the $\mathrm{BCCH}$ carrier and the estimation of $f_{\mathrm{o}}$ in order to tune the oscillator in the receiver.

\section{FREQUENCY BURST DETECTION}

In this section, we characterize two promising algorithms for FB detection which exploit different signal characteristics for their detection statistics. The proposed phase variance detection (PVD) algorithm and the LRP algorithm [6] are suitable to be combined with FOE, in order to realize a lowcomplexity frequency synchronizer solution for GSM-enabled receivers.

The PVD algorithm exploits the characteristic of the phase course of the complex sinusoid transmitted on FBs. The unwrapped phase follows a linear ramp whose inclination is proportional to the frequency of the signal. The difference of the phases of two consecutive symbols in a FB is assumed to be constant in a noise-less case. Therefore, the variance of the phase differences can be used as a reliable metric for FBD. To this end, the proposed algorithm first collects a vector $\boldsymbol{\Delta} \phi=\left[\Delta \phi_{i}, \ldots, \Delta \phi_{i-N_{\mathrm{DET}}}\right]$ containing $N_{\mathrm{DET}}$ phase differences of subsequent symbols $\Delta \phi_{i}=\angle y[n]-\angle y[n-1]$. Subsequently we compute the variance

$$
\hat{\sigma}^{2}=\hat{E}\left[\boldsymbol{\Delta} \phi^{2}\right]-\hat{E}[\boldsymbol{\Delta} \phi]^{2},
$$

where $\hat{E}[\cdot]$ denotes the empirically estimated expectation value and $\angle(\cdot)$ the phase of a complex symbol. If the estimated variance $\hat{\sigma}^{2}$ in (2) is below a threshold value, the start of a FB is detected at sample index $i-N_{\mathrm{DET}}$.
The LRP detector uses the absolute value of the signal's autocorrelation as decision statistic:

$$
\left|r_{L}\right|=\left|E\left\{y_{n} y_{n-L}^{*}\right\}\right|
$$

If $\left|r_{L}\right|$ exceeds a certain threshold, a FB is detected. The correlation sequence $r_{L}$ is estimated as follows:

$$
\hat{r}_{L}=\sum_{n=1}^{N_{\mathrm{DET}}} y_{n} y_{n-L}^{*}
$$

The two algorithms under consideration, PVD and LRP, are compared with a standard filter-based approach. Such detectors place a bandpass filter around $f_{\text {sym }} / 4$ (e.g., a 2 nd order IIR notch filter [7], herein referred as NOT) and use power measurements at filter input and output as decision statistic for detection. The power ratio between filter input and output is then compared to a certain threshold, in order to determine whether a FB has been found.

\section{A. Performance and Complexity Comparison}

The algorithms are characterized in terms of detection probability $P_{\mathrm{D}}$ and false alarm probability $P_{\mathrm{F}} . P_{\mathrm{D}}$ is the probability that the detector identifies a FB correctly among non-FB bursts (e.g., a burst which contains data traffic), and $P_{\mathrm{F}}$ is the probability that a non-FB is detected as a FB. All three approaches compare their decision statistic to a certain threshold, which determines $P_{\mathrm{D}}$ and $P_{\mathrm{F}}$. For GSM synchronization $P_{\mathrm{F}}$ needs to be significantly lower than the probability $1-P_{\mathrm{D}}$ that a FB has not been detected, because only about $1.25 \%$ of the bursts on BCCH are FBs (cf. Section II).

In order to find a robust solution for FBD, the algorithms have been configured to achieve a false alarm rate $P_{\mathrm{F}}$ of $0.1 \%$ under demanding conditions. To this end, we evaluate detection performance with worst-case assumptions, i.e., for very low SNR and for a frequency offset range of $\pm 18 \mathrm{kHz}$. With fixed $P_{\mathrm{F}}$ the threshold value can be determined for each specific algorithm, resulting in the corresponding $P_{\mathrm{D}}$.

In order to minimize synchronization time in GSM, we aim at $\mathrm{FB}$ detection and FOE on the same FB without expensive buffering of received I-/Q-samples. To this end, we perform FBD on the first part of the FB, such that the remaining samples can be used for FOE. Simulations have shown, that using approximately one third of $N_{\mathrm{TOT}}$, i.e., $N_{\mathrm{DET}}=48$ data samples for detection, is sufficient to meet above constraints.

Computer simulations for detector performance evaluation were assessed on a standard compliant GSM/EGPRS2 framework. The detection probability was evaluated for the specified multipath-fading channel profiles for $250 \mathrm{BCCH}$ multiframes, each containing 51 TDMA frames [8]. The simulation results shown in this paper have been achieved by applying a onetap channel, which simplifies the illustration and comparison to other work, and which allows us to compare the simulated curves with the measurement results of the testbed, where a direct radio link is set up (cf. Section V). Figure 1 shows $P_{\mathrm{D}}$ as a function of the SNR for a frequency offset of $f_{\mathrm{o}}=7.2 \mathrm{kHz}$ as an example. 
The bandwidth of the notch filter is set according to [7], where the parameters of the NOT approach have been optimized for GSM. The fundamental parameter in the LRP detector is the correlation lag $L$, which trades $P_{\mathrm{D}}$ against $P_{\mathrm{F}}$. Simulations have shown that for a fixed $P_{\mathrm{F}}$ of $0.1 \%$ the maximum $P_{\mathrm{D}}$ can be achieved with $L=2$. In order to reduce computational complexity, (3) can be computed by using the $\mathcal{L}^{1}$-norm instead of the optimum $\mathcal{L}^{2}$-norm. The optimum correlation lag when using the $\mathcal{L}^{1}$-norm has been found to be $L=3$. This approximation leads to a negligible performance loss, as can be seen in Figure 1. Instead, choosing a suboptimum correlation lag, e.g. $L=1$, can lead to significantly lower $P_{\mathrm{D}}$.

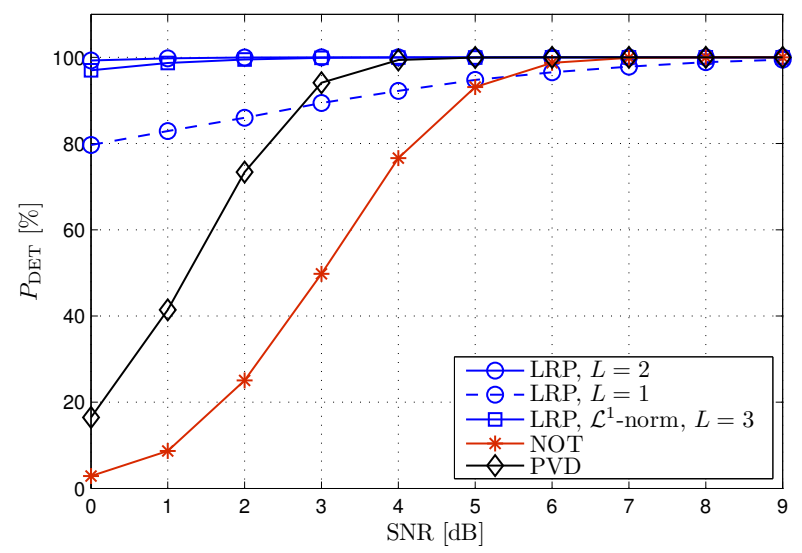

Fig. 1. Detection probability $P_{\mathrm{D}}$ of PVD, NOT and LRP at $f_{\mathrm{o}}=7.2 \mathrm{kHz}$.

The notch filter approach shows poor performance compared to the other two approaches, since the attenuation of the notch filter is not strong enough to provide an unambiguous ratio of input and output power as decision metric ${ }^{3}$. The PVD algorithm's detection probability is the best for SNR regimes above $4 \mathrm{~dB}$, but decays rapidly below $4 \mathrm{~dB}$. The FBD with LRP shows best detector performance in low SNR regime. Contrary to NOT, PVD and LRP show independent detection performance over the entire frequency offset range of $f_{\mathrm{o}}= \pm 18 \mathrm{kHz}$. For typical SNR-values in modern GSM receivers (i.e., $\mathrm{SNR} \geq 7 \mathrm{~dB}$ ), all three algorithms provide very high detection probability of $P_{\mathrm{D}} \geq 99.9 \%$.

Table I shows the complexity of the three detection algorithms given in number of additions, multiplications, and phase-calculations per symbol required for the FBD. As can be seen, the computational complexity of the notch based FB detector is highest, since it requires 6 multiplications of complex-values samples with real-valued coefficients and 6 complex-valued additions for the 3-tap IIR filtering operation. In addition, 4 real-valued multiplications and additions are required for the computation of the signal power before and after filtering. The LRP approach requires a costly square root operation for computing the absolute value in (3), which can be

\footnotetext{
${ }^{3}$ Note that NOT's performance strongly depends on the frequency offset. E.g., for $f_{\mathrm{o}}$ close to $f_{\mathrm{sym}} / 4$ NOT performs significantly better.
}

avoided by using the $\mathcal{L}^{1}$-norm (see above). We conclude that the complexity of PVD and LRP with $\mathcal{L}^{1}$-norm is significantly lower compared to standard filter-based approaches.

TABLE I

COMPARISON OF OPERATIONS/SYMBOL IN FBD

\begin{tabular}{|c|c|c|c|}
\hline & LRP $^{a}$ & PVD & NOT \\
\hline Multiplications $^{b}$ & 4 & 2 & 16 \\
\hline Additions $^{b}$ & 4 & 3 & 16 \\
\hline Phase calculations $^{2}$ & 0 & 1 & 0 \\
\hline
\end{tabular}

${ }^{a}$ LRP algorithm with $\mathcal{L}^{1}$-norm for the computation in (3).

${ }^{b}$ The table shows the number of real-valued operations (complex-valued multiplications are counted as 4 real-valued multiplications and 2 additions).

\section{FREQUENCY OFFSET ESTIMATION}

Estimating the frequency of a single sinusoid under the influence of noise is a classical parameter estimation problem. Besides the optimal maximum likelihood approach [9], famous estimators which achieve performances up to the Cramer Rao lower bound (CRLB) have been proposed.

Periodogram estimators (e.g., [9]) can achieve close-tooptimum accuracy at the cost of high computational complexity. Instead, algorithms based on phases or phases of autocorrelations are suited for low-complexity hardware implementations. We propose to further reduce implementation complexity of these algorithms by re-using results of calculations in the FBD. In this section, we describe two FOE approaches that can be implemented efficiently by exploiting the results of PVD and LRP detection (cf. Section III).

Kay's weighted phase averager (WPA) [10], where the unwrapped phase is fitted to a straight line, requires the costly computation of phase differences. By combining the WPA with PVD, the phase differences $\boldsymbol{\Delta} \phi=\left[\Delta \phi_{i}, \ldots, \Delta \phi_{i-N_{\mathrm{DET}}}\right]$ required for FB detection can be re-used for FOE. Alternatively, Kay's Weighted linear predictor (WLP) [10], where the phases of autocorrelations $\angle r_{L}$ are used for FOE, can be combined well with the LRP detector. Results of the correlation in (3) can be re-used for FOE.

Unfortunately, both WPA and WLP estimation algorithms can not achieve the desired accuracy of $0.1 \mathrm{ppm}(90 \mathrm{~Hz}$ at the lower carrier frequency of $900 \mathrm{MHz}$ ) in the SNR-range of practical interest for modern GSM receivers, i.e., $S N R \geq 7 \mathrm{~dB}$. The mean frequency estimation error of both algorithms is shown in Figure 2. It can be seen that even increasing the number of considered symbols to $N_{\text {TOT }}$ would not lead to the required performance.

The FOE with WLP uses a correlation lag of $L=1$ which limits the estimation performance. Choosing a correlation lag $L>1$ improves the estimation performance significantly. The optimal relation of $L$ to the amount of input samples $N_{\mathrm{EST}}$ is given by $L=2 N_{\mathrm{EST}} / 3$ in order to achieve best FOE accuracy for high SNR [6]. The drawback of using an $L>1$ is the introduced frequency ambiguity since the phase course may experience more than one wrap. In this case, the estimated frequency offset $\hat{\omega}$ in (5), with $T_{S}$ denoting the 


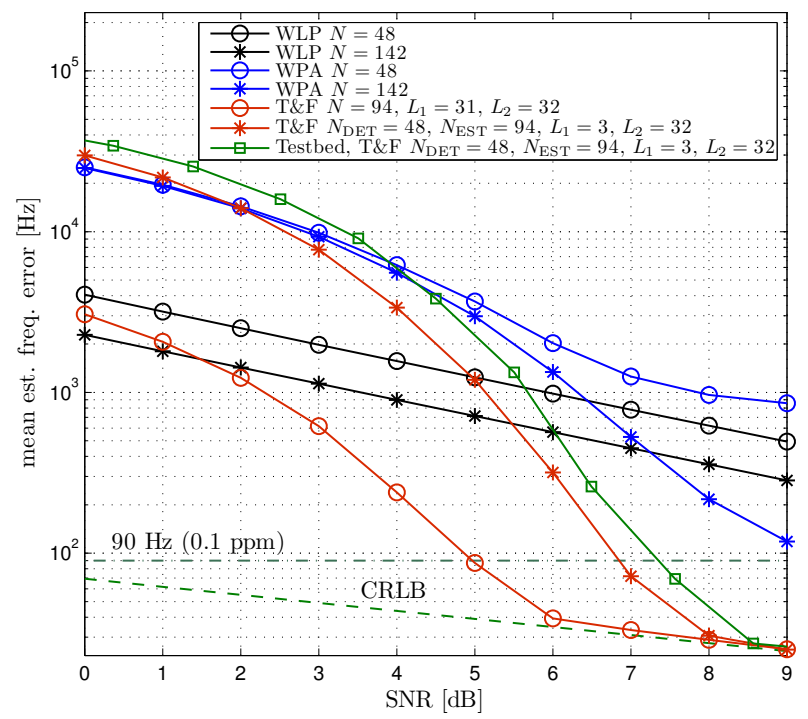

Fig. 2. Mean $f_{\mathrm{o}}$ estimation error of WPA and T\&A settings over SNR.

sampling period, has multiple solutions, since the number of phase wraps $l \in\{0, \ldots, L\}$ is unknown.

$$
\hat{\omega}=\frac{\angle r_{L}+2 \pi l}{L T_{\mathrm{S}}}
$$

The T\&F estimator [5] resolves this ambiguity without being computationally intensive by computing two correlations $r_{L_{1}}$ and $r_{L_{2}}$ with different correlation lags $L_{1}$ and $L_{2}$. If $L_{1}$ and $L_{2}$ are relatively prime, unambiguous FOE up to $f_{\mathrm{o}}^{\max }=67.7 \mathrm{kHz}$. is possible.

The choice of $L_{1}$ and $L_{2}$ and $N$ affects the performance of the T\&F algorithm. Choosing $L_{2}=L_{1}+1$ has been proposed in [5] as a common setting. Our analyses have shown that ambiguity resolution benefits from a small correlation lag, whereas high frequency accuracy can only be achieved with a larger lag. Therefore, we propose the computation of the correlation sequences $r_{L_{1}}$ and $r_{L_{2}}$ with $L_{1}<<L_{2}$, which allows good ambiguity resolution due to small $L_{1}$ and high frequency accuracy due to large $L_{2}$. Furthermore, we propose to use different numbers of samples $N_{1}$ and $N_{2}$ for the computation of $r_{L_{1}}$ and $r_{L_{2}}$, to optimize the algorithm for the needs of combined low-complexity frequency synchronization.

In particular, in order to be able to re-use the results of the correlation sequence of the LRP detector with $\mathcal{L}^{1}$-norm, we set $L_{1}=3$ and $N_{1}=N_{\mathrm{DET}}=48$ (cf. Section III)). The second lag is set to $L_{2}=32$ to obtain a highly accurate estimate for $f_{\mathrm{o}}$ in the high SNR regime, and $N_{2}$ results from the remaining samples after FBD: $N_{2}=N_{\text {TOT }}-N_{\text {DET }}=94$.

As can be seen in Figure 2, our T\&F settings allow for close-to-optimum frequency offset estimates in high SNR regimes, and for the required $0.1 \mathrm{ppm}$ accuracy for $\mathrm{SNR} \geq 7 \mathrm{~dB}$. Increasing the number of samples $N_{1}$ used for the $r_{L_{1}}$ computation or using an even smaller correlation lag $L_{1}$ improves the ambiguity resolution, and therefore leads to better results for low SNR. However, our parameter settings are best for the combined FB detection and FOE on a single FB: we achieve high detector performance at low complexity by using the LRP with $\mathcal{L}^{1}$-norm on the first $N_{\text {DET }}$ samples, and we achieve the required frequency accuracy in the SNR range of practical interest for modern GSM receivers by using the remaining $N_{\text {EST }}$ samples for FOE.

Regarding complexity, the original $\mathrm{T} \& \mathrm{~F}$ algorithm requires $Q=2 N-L_{1}-L_{2}$ complex-valued multiplications and additions, and 2 phase calculations per frequency estimate. By using different $N_{\mathrm{DET}}$ and $N_{\mathrm{EST}}$ for the computation of $r_{L_{1}}$ and $r_{L_{2}}$, the number of computations is given according to $Q=N_{\mathrm{DET}}+N_{\mathrm{EST}}-L_{1}-L_{2}$. By re-using the results of the LRP detection, $Q$ can be reduced to $Q=N_{\mathrm{EST}}-L_{2}$, which results in about half the computational complexity compared to standard T\&F with conventional parameters [5]. For comparison, the periodogram estimator which takes the peak of the spectra requires as many as $6 N \log _{2} N$ real-valued multiplications for the radix-2 FFT [9].

We conclude that better FOE performance can be achieved with periodogram estimators or conventional $\mathrm{T} \& \mathrm{~F}$ algorithm, however, the proposed $\mathrm{T} \& \mathrm{~F}$ with optimized parameter settings meets the requirements of state-of-the-art GSM receivers at lowest complexity.

\section{HARDWARE IMPLEMENTATION}

For functional verification of algorithm hardware implementations and for measurements in real-world conditions we have assembled a GSM/EGPRS2 testbed setup. The corresponding block-diagram is shown in Figure 3. The performance of the FBD implementation can be measured by using a standard compliant BTS emulator to generate and transmit $\mathrm{BCCH}$ multiframes. The BTS emulator in our setup comprises of OpenBTS ${ }^{4}$ [11] and GNU radio software running on computer \#1, and a USRP board with antenna to transmit the signal over the air to the TRX support board. In order to measure the performance of our FOE implementation, a signal generator provides a sinusoid $67.7 \mathrm{kHz}$ above the carrier frequency, which corresponds to a continuous stream of frequency bursts. The signal power of the sinusoid is configurable which allows FOE measurements over SNR.

The received signal on the TRX support board is fed into a state-of-the-art RF transceiver $\mathrm{IC}^{5}$ to down-convert and digitize the received signal before the I/Q samples of the complex baseband enter the Spartan3 FPGA at fourfold oversampling. The samples are directly fed to an on-board digital-to-analog converter (DAC), which is connected via RF cable to a spectrum analyzer for debugging purposes. The received samples are also read by computer \#2 via high-speed parallel port (HSPP), which is also used to configure the RF TRX chip via Spartan3 FPGA. Furthermore, the samples are

\footnotetext{
${ }^{4}$ OpenBTS is an open-source BTS software, using the Universal Software Radio Peripheral (USRP) board to establish a GSM air interface to MSs.

${ }^{5}$ IRIS305 from ACP AG: a single-chip RF transceiver supporting TDSCDMA/HSPA (3G) and GSM/EGPRS2 (2G/2.75G).
} 


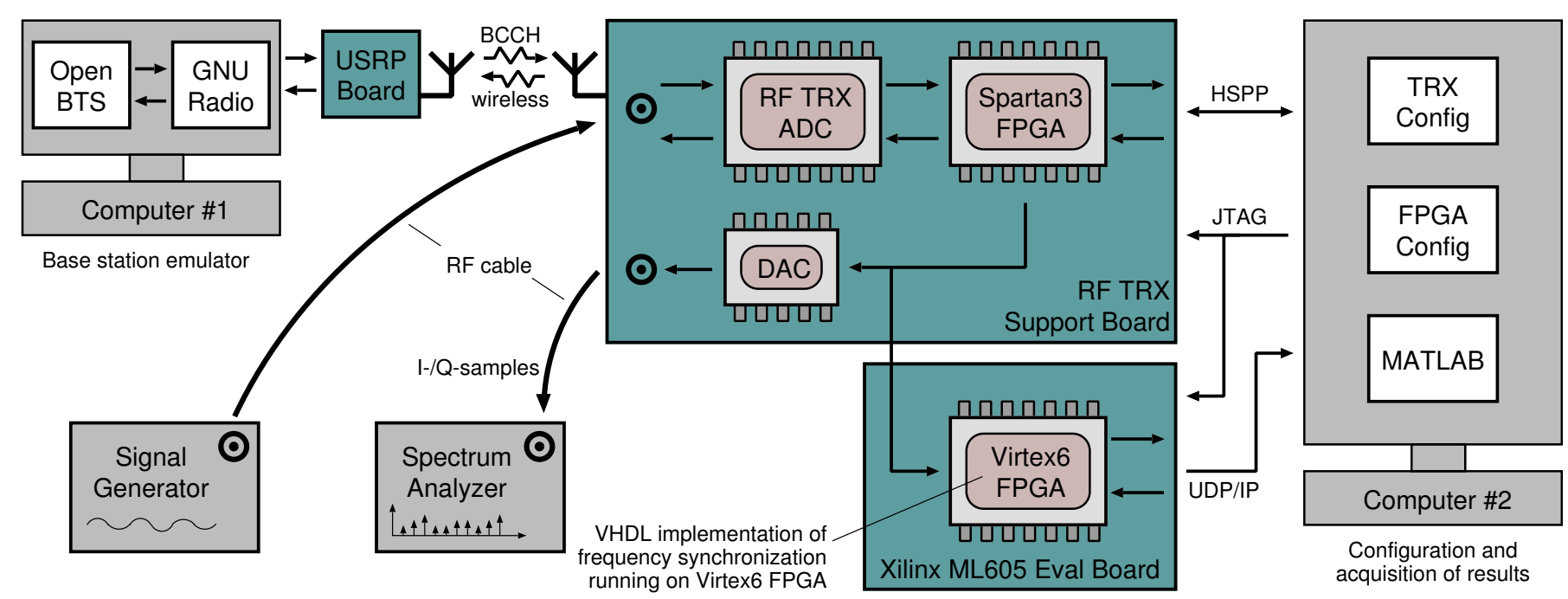

Fig. 3. Real time GSM/EGPRS2 testbed setup.

fed via parallel port to the Virtex6 FPGA on a Xilinx ML605 evaluation board, where our digital baseband signal processing solutions for GSM/EGRPS2 are implemented and verified. Measurement results are sent from Virtex6 FPGA directly to MATLAB for post-processing and visualization on computer \#2 via UDP/IP.

For the purpose of this paper, on Virtex6 FPGA we have implemented a digital front-end (DFE) with DC-offset removal and decimation filtering, in order to obtain the DC-free received signal at symbol rate, as required by the FBD and FOE algorithms. We have developed a low-complexity hardware architecture for our frequency synchronization solution for GSM and mapped the corresponding VHDL code also to the Virtex6 FPGA ${ }^{6}$. We have successfully verified the functionality of the implementation. Measurement results of the FOE with combined LRP and T\&F (with the proposed setting in Section IV) are shown in Figure 2 and prove the simulation results. The measurements of our hardware implementation with our real-world environment testbed, including RF TRX, $\mathrm{ADC}$, and $\mathrm{DFE}$, are within $0.5 \mathrm{~dB}$ compared to the ideal floating-point curves simulated with MATLAB.

In order to verify the functionality of the system, we realized an open-loop frequency offset correction: our implementation estimates the frequency offset and transmits the result via UDP/IP from the Virtex6 FPGA to computer \#2. The estimated frequency offset is used to re-configure the DCXO of the RF transceiver via HSPP. Figure 4 shows the sequence of events: the red dots mark the time instances where a new frequency is applied at transmit side. Directly afterwards, our FOE realization computes a new $f_{\mathrm{o}}$ value, as can be seen in the blue curve. An arbitrarily chosen number of time instances later (20 in this case, for the sake of clarity), an oscillator tuning word is sent from computer \#2 to the RF transceiver, in order to tune the DCXO according to the frequency offset

${ }^{6}$ Note that we have developed other critical signal-processing blocks for the GSM/EGPRS2 baseband (cf [3]), that can be mapped to our receiver testbed. estimation $f_{\mathrm{o}}$. The tuning word, marked with green dots, configures the corrected (absolute) carrier frequency of the RF transceiver. Immediately after the DCXO has been tuned, the estimated frequency offset reduces to $f_{\mathrm{o}} \approx 0$, which shows that frequency synchronization is achieved.

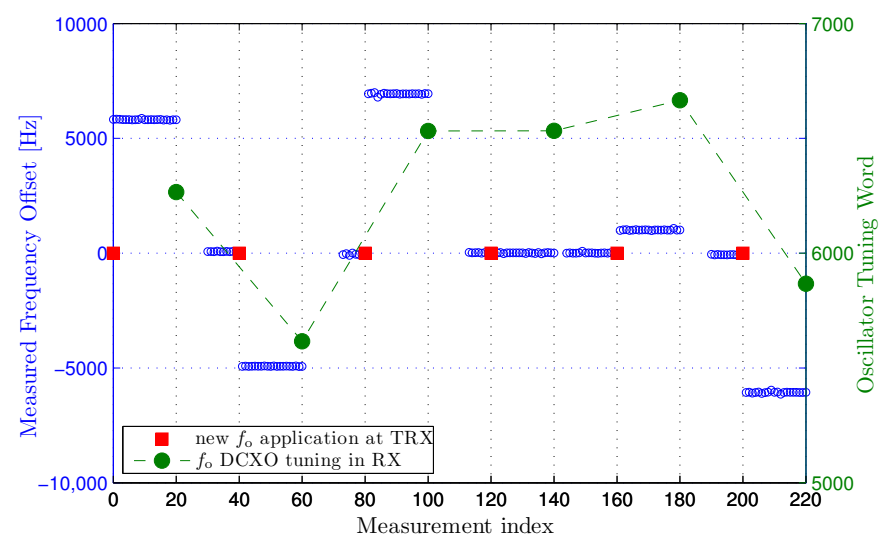

Fig. 4. Open loop frequency offset correction. The time interval between two measurement indices is $8 \mathrm{~ms}$.

\section{CONCLUSION}

The proposed combination of LRP and T\&F algorithms for frequency burst detection and frequency offset estimation is highly suitable for fast and reliable synchronization in modern GSM receivers. We have optimized the algorithm parameters that even in low-SNR regimes and for high frequency offset, frequency bursts can be detected reliably and frequency can be estimated accurately. The hardware implementation on a GSM/EGPRS2 testbed and corresponding measurements with standard-compliant GSM-signals prove the functionality and feasibility of our proposed frequency synchronization concept in real systems. 


\section{ACKNOWLEDGMENT}

This work was funded by CTI, Switzerland (project no 11370.1 PFNM-NM) in collaboration with Advanced Circuit Pursuit (ACP) AG. We thank ACP AG for providing us the IRIS305 single-chip RF transceiver for our testbed setup. In addition we want to thank David Tschopp for his support on the testbed and Pirmin Vogel for his valuable work during his student project.

\section{REFERENCES}

[1] "GSM Association, Market Data, www.gsmworld.com, August 2010."

[2] A. Cicalini et al., "A $65 \mathrm{~nm} \mathrm{cmos}$ SoC with embedded hsdpa/edge transceiver, digital baseband and multimedia processor," in Solid-State Circuits Conference Digest of Technical Papers (ISSCC), 2011 IEEE International, feb. 2011, pp. $368-370$.

[3] C. Benkeser, A. Bubenhofer, and Q. Huang, "A 4.5mW Digital Baseband Receiver for Level-A Evolved EDGE," in Solid-State Circuits Conference Digest of Technical Papers (ISSCC), 2010 IEEE International, feb 2010, pp. 276-277.
[4] 3GPP TR 45.010: GSM/EDGE Radio Access Network; Radio subsystem synchronization, TS 45.010, Rev. 9.0.0, Nov. 2009.

[5] D. Tufts and P. Fiore, "Simple, effective estimation of frequency based on prony's method," in Acoustics, Speech, and Signal Processing, ICASSP Conference Proceedings, IEEE International Conference on, vol. 5, may 1996, pp. 2801-2804.

[6] G. Lank, I. Reed, and G. Pollon, "A semicoherent detection and doppler estimation statistic," Aerospace and Electronic Systems, IEEE Transactions on, no. 2, pp. 151-165, 1973.

[7] U. Jha, "Acquisition of frequency synchronization for gsm and its evolution systems," in Personal Wireless Communications, 2000 IEEE International Conference on. IEEE, 2000, pp. 558-562.

[8] 3GPP TR 45.001 GSM/EDGE Radio Access Network; Physical layer on the radio path; General description, TS 45.001, Rev. 9.1.0, Nov. 2009.

[9] D. Rife and R. Boorstyn, "Single tone parameter estimation from discrete-time observations," Information Theory, IEEE Transactions on, vol. 20, no. 5, pp. 591-598, Sep. 1974.

[10] S. Kay, "Statistically/computationally efficient frequency estimation," in Acoustics, Speech, and Signal Processing, ICASSP, International Conference on, vol. 4, Apr. 1988, pp. 2292-2295.

[11] "OpenBTS, http://openbts.sourceforge.net, January 2011." 